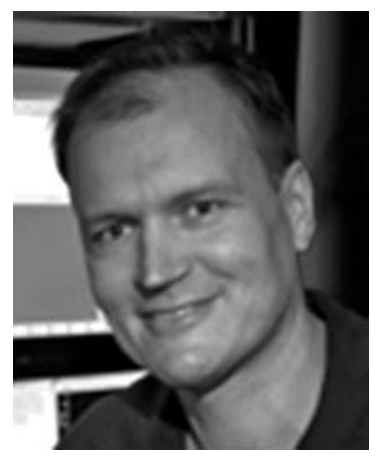

\title{
A Conversation with Karel Svoboda
}

\author{
InTERVIEWER: KaREN CARNIOL \\ Deputy Editor, Cell
}

Karel Svoboda is a Group Leader at Howard Hughes Medical Institute's Janelia Farm Research Campus.

Karen Carniol: One of the many things you're interested in is understanding the neural coding that underlies object recognition and decision-making. One of the ways you've chosen to investigate that is through tasks with rodents using their whiskers to perceive. What are you learning?

Dr. Svoboda: Mice and other rodents use their whiskers to explore their environment. It seems like a weird system to study, rather than vision or touch through their paws, but it turns out to have some wonderful advantages and wonderful features for detailed mechanistic analysis of basic cognitive processes, like active sensation, perception, and decision-making. One of the key features is that the system is exquisitely mapped all the way into the cortex. There's a one-to-one correspondence between individual whiskers and small groups of neurons that process information from that whisker. We can zoom in to the relevant neurons. We can record and manipulate the relevant neurons, ask how manipulations have an impact on behavior and be very quantitative about that.

In many other systems, that kind of study is a needle in a haystack problem. Here we know where to look.

The barrel system first had its heyday in studies of experience-dependent plasticity. As the animal develops, information processing is restricted to individual barrels. Beyond that, there are a lot of features in the response of individual neurons to the dynamics of individual whiskers that are learned. Different neurons show different rates of adaptation. In this system we can have a comprehensive understanding of what the neural population is doing that processes information from one whisker.

Karen Carniol: What's the route from understanding the somatosensory perception to a decision-making process in that system?

Dr. Svoboda: We interact with the world by sweeping our eyes over objects or visual scenes to extract the information we want. Through visual search we produce input to the visual system. But the visual system is never studied that way, because it's very difficult to keep track of what exactly a monkey or a mouse looked at. In the whisker system, we can study the decision-making in the barrel cortex in the context of active sensation where the mouse moves its whisker to explore an object, to touch it, to palpate it, to learn about its location or its shape, and so on and so forth.

You can ask questions about what is encoded in the barrel cortex. You can ask about neural coding. Information is carried by action potentials, or spikes, and you can ask questions about how action potentials, trains of action potentials, in different neurons encode information about where the whisker is, what the whisker touches, how the whisker is touched, how it is informed. Then you can go one step further and actually manipulate the activity, to ask not only what is encoded, but what the rest of the brain reads out from that activity, something you can do in this system that is very difficult to do in other systems.

Karen Carniol: What are some of the techniques that you've developed?

Dr. Svoboda: We use electrophysiology extensively to learn what is encoded in the brain. So, what kind of tactile features, what specific types of neurons there are in the barrel cortex. Remember each barrel is still a complex structure with about 10,000 neurons, and at least 20 cell types. We can ask what is encoded in specific neurons using electrophysiology and then with closed loop behavior and photostimulation using optogenetics we can then manipulate these action potential trains in a very precise manner to see if we can push perception one way or another. That is really the arbiter of truth about what actually is extracted about the world by the animal from these action potential trains.

Karen Carniol: The action potentials lead me to my next question, which is another thing you've been looking at: individual synapses, their plasticity, and their stability. Can you talk about that?

Dr. Svoboda: This is an area of study that has been on the wane in the laboratory. Even though we published a paper this year, that experiment was probably done 5 or 6 years ago. The experiments you refer to really go back to my 
work at Cold Spring Harbor starting in the late 1900s. We set up some very powerful microscopes that allow us to look at individual synapses in the intact brain. One of the things that had always puzzled me was this literature on enrichment-related plasticity, structural plasticity, of neurons. We know that memories last for a long time, so I thought it was very important to study how stable neurons are, how stable synapses are, what is the lifetime of synapses, or different types of synapses. That's how we got to study structural plasticity in the adult brain and its relationship to experiential manipulations.

What we found was that a substantial fraction of synapses, at least in nonpathological conditions, are remarkably stable. They can last for many months, in some cases for the lifetime of an animal, which is something that I find quite surprising because they are made up of small numbers of fragile, labile proteins. It's still a mystery how that works. But then another fraction of synapses turn over, they appear, and disappear and that is modulated by experience. It's also cell-type-specific. It happens at different rates, and different synapses, thalamocortical synapses, input to the cortex tend to be more stable than intracortical synapses and so on and so forth.
This study of structural plasticity and the cell-type-specific effects really got me very much interested in cortical circuits and how they work, because we could not put this into a functional context without really knowing more about how neurons are hooked up. The logic of information processing is in cortical circuits.

Karen Carniol: Do you see the field of neural circuits and synaptic molecular biology converging at some point in the future?

Dr. Svoboda: I absolutely do and I have a vision of coming back to it. I've dabbled in studying learning at the level of circuits. I've learned that learning is a very, very difficult problem, so we've stepped back and study what I would call function. I want to go back and learn and develop good learning paradigms, for example, good motor learning paradigms and then go in and dissect, look for engrams at the level of defined synaptic pathways. We have to be able to show that those pathways and these changes at synapses are causally related to the memory, which is something that we may be able to get a handle on using new molecular methods over the next decade or so. 


\section{$\$_{\text {CSH }}^{\infty}$ Cold Spring Harbor Symposia SYMPOSIA On Quantitative Biology}

\section{A Conversation with Karel Svoboda}

Cold Spring Harb Symp Quant Biol 2014 79: 293-294

Access the most recent version at doi:10.1101/sqb.2014.79.17

\section{License}

Email Alerting Receive free email alerts when new articles cite this article - sign up in Service the box at the top right corner of the article or click here. 